Research paper

\title{
Optimization and validation of a neutralizing antibody assay for HIV-1 in A3R5 cells
}

\author{
Marcella Sarzotti-Kelsoe ${ }^{\mathrm{a}, \mathrm{b}, \mathrm{d}, *}$, Xiaoju Daniell $^{\mathrm{b}}$, Christopher A. Todd ${ }^{\mathrm{b}}$, Miroslawa Bilska ${ }^{\mathrm{b}}$, \\ Amanda Martelli ${ }^{\mathrm{b}}$, Celia LaBranche ${ }^{\mathrm{b}}$, Lautaro G. Perez ${ }^{\mathrm{b}}$, Christina Ochsenbauer ${ }^{\mathrm{c}}$, John C. Kappes ${ }^{\mathrm{c}}$, \\ Wes Rountree $^{\mathrm{d}}$, Thomas N. Denny ${ }^{\mathrm{d}}$, David C. Montefiori ${ }^{\mathrm{b}}$ \\ a Department of Immunology, Duke University Medical Center, Durham, NC, USA \\ b Department of Surgery, Duke University Medical Center, Durham, NC, USA \\ c Department of Medicine, University of Alabama at Birmingham, Birmingham, AL, USA \\ ${ }^{\mathrm{d}}$ Duke Human Vaccine Institute, Durham, NC, USA
}

\section{A R T I C L E I N F O}

\section{Article history:}

Received 23 September 2013

Received in revised form 22 January 2014

Accepted 26 February 2014

Available online $\mathrm{xxxx}$

\section{Keywords:}

Neutralizing antibody

Assay validation

HIV

A3R5 cells

\begin{abstract}
A B S T R A C T
A3R5 is a human CD4 ${ }^{+}$lymphoblastoid cell line that was engineered to express CCR5 and is useful for the detection of weak neutralizing antibody responses against tier 2 strains of HIV-1. Here we describe the optimization and validation of the HIV-1 neutralizing antibody assay that utilizes A3R5 cells, performed in compliance with Good Clinical Laboratory Practice (GCLP) guidelines. The assay utilizes Renilla luciferase-expressing replication competent infectious molecular clones (IMC) encoding heterologous env genes from different HIV-1 clades. Key assay validation parameters tested included specificity, accuracy, precision, limit of detection and quantitation, specificity, linearity and range, and robustness. Plasma samples demonstrated higher non-specific activity than serum samples in the A3R5 assay. This assay can tolerate a wide range of virus input but is more sensitive to cell concentration. The higher sensitivity of the A3R5 assay in neutralization responses to tier 2 strains of HIV-1 makes it complementary to, but not a substitute for the TZM-bl assay. The validated A3R5 assay is employed as an endpoint immunogenicity test for vaccine-elicited neutralizing antibodies against tier 2 strains of HIV-1, and to identify correlates of protection in HIV-1 vaccine trials conducted globally.
\end{abstract}

(c) 2014 Elsevier B.V. All rights reserved.

\section{Introduction}

Standardized assessments of the neutralizing antibody response are a critical component of the vaccine discovery process. In the case of HIV-1 vaccines, large numbers of samples will need to be assayed against multiple strains of virus in different laboratories. Because no single assay is capable of detecting all neutralizing antibodies against HIV-1, it has been recommended that multiple assays be used when evaluating

\footnotetext{
* Corresponding author at: Duke University Medical Center, 2812 Erwin Rd., Suite 301, Erwin Terrace II, Durham, NC 27705, USA. Tel.: + 1919684 6373; fax: +19196817766.
}

E-mail address: marcella.sarzottikelsoe@dm.duke.edu (M. Sarzotti-Kelsoe). vaccine-elicited neutralizing antibody responses (Polonis et al., 2008; Fenyo et al., 2009). Advances in assay technology have led to major improvements in how HIV-1 neutralizing antibodies are measured.

Two high throughput assays using cell lines as the target for HIV-1 infection, and luminescence as the readout of target cells' viral infection have been developed: the TZM-bl assay (Wei et al., 2002, 2003; Montefiori, 2009), which was formally validated (Sarzotti-Kelsoe et al., 2013), and more recently, the A3R5 assay (McLinden et al., 2013). Genetic variability has given rise to a multitude of HIV-1 variants that differ in their overall neutralization phenotype. Thus, HIV-1 variants that exhibit a high level of neutralization susceptibility to HIV-1-positive sera are classified as tier 1 viruses 
(Seaman et al., 2010). Most circulating strains have evolved under immune pressure to exhibit an overall lower level of neutralization susceptibility that is classified as a tier 2 phenotype (Seaman et al., 2010). Neutralization of tier 2 viruses is generally considered a priority for vaccines (Mascola et al., 2005).

Neutralizing antibodies against tier 2 strains of HIV-1 can be efficiently measured in A3R5 cells after infection with Renilla luciferase (LucR)-expressing replication competent infectious molecular clones (IMC) encoding heterologous env genes from different HIV-1 clades (collectively referred to as Env.IMC.LucR viruses) (Ochsenbauer and Kappes, 2009; Edmonds et al., 2010; Montefiori et al., 2012; Ochsenbauer et al., 2012; McLinden et al., 2013). Although the TZM-bl assay is used worldwide and may be considered the "gold standard assay" to detect neutralization of tier 1 and tier viruses (Ozaki et al., 2012; Todd et al., 2012; Seaman et al., 2010), the A3R5 assay is a new assay that demonstrates greater sensitivity in the detection of neutralizing antibody responses against tier 2 viruses. The A3R5 assay is complementary to, but not a substitute for, the TZM-bl assay.

In these studies, the A3R5 assay was optimized and validated in Good Clinical Laboratory Practices (GCLP) compliance (Stiles et al., 2003; Ezzelle et al., 2008; SarzottiKelsoe et al., 2009). Validating an assay consists of analyzing the assay parameters recommended by the International Conference on Harmonisation (ICH)-Q2 (R1) guidelines (I.C.H. Guideline, 2005): specificity, accuracy, precision, detection and quantitation limits, linearity, range, and robustness. The process began with numerous optimization experiments to help establish pre-set acceptance criteria for the formal validation experiments. Applicable ICH parameters of validation were selected and a validation plan, inclusive of a statistical analysis plan, was written and authorized by the Duke Center for AIDS Research Central Quality Assurance Unit (CQAU). The assay validation was performed, data statistically analyzed, and a validation report was written and approved (see schematic in Todd et al., 2013).

\section{Materials and methods}

Many of the methods described in this report have been reported elsewhere (Montefiori, 2009; Montefiori et al., 2012; McLinden et al., 2013). This report describes the key elements that went into the formal optimization and validation of the A3R5 assay and is not meant to be an exhaustive list of all optimization and validation experiments that have been performed.

\subsection{Cell lines}

The A3.01/R5.7 (A3R5) cell line was derived from the A3.01 human lymphoblastoid cell line that naturally expresses CD4 and CXCR4 (Folks et al., 1985) and was engineered to express CCR5 (McLinden et al., 2013) (NIH AIDS Reagent Repository Program \#12386). These cells were maintained in culture for a maximum of three months. The TZM-bl cell line was derived from a HeLa cell clone that was engineered to express CD4, CCR5 and CXCR4 (Platt et al., 1998) and to contain integrated reporter genes for firefly Luc and Escherichia coli $\beta$-galactosidase under the control of an HIV-1 long terminal repeat (Wei et al., 2002) (NIH AIDS Reagent Repository Program \#8129). 293T/17 cells were obtained from the American Tissue Culture Collection (catalog no. 11268).

\subsection{Culture conditions}

The A3R5 cell line was maintained in Roswell Park Memorial Institute (RPMI) Medium with L-glutamine, $25 \mathrm{mM}$ HEPES (4-(hydroxyethyl)-1-piperazineethanesulfonic acid) (Gibco BRL Life Technologies) containing 10\% heat-inactivated fetal bovine serum (FBS) and $50 \mu \mathrm{g} / \mathrm{ml}$ gentamicin/ml (hereafter referred to as RPMI Growth Medium) in vented T-75 culture flasks (Corning Costar). $1 \mathrm{mg} / \mathrm{ml}$ of Geneticin (G418) (Gibco BRL, Life Technologies) was added to the flask containing A3R5 cells. Cell cultures were split 1:10 when they reached a density of approximately $1.5 \times 10^{6}$ cells $/ \mathrm{ml}$. The $293 \mathrm{~T} / 17$ cell line was maintained in Dulbecco's Modified Eagle's Medium (DMEM) with L-glutamine, sodium pyruvate, glucose, pyridoxine and $25 \mathrm{mM}$ HEPES (Gibco BRL Life Technologies) containing 10\% heat-inactivated FBS and $50 \mu \mathrm{g}$ gentamicin/ml (hereafter referred to as DMEM Growth Medium) in vented T-75 culture flasks (Corning Costar). Cell monolayers were split 1:10 at confluence by treatment with $0.25 \%$ trypsin, $1 \mathrm{mM}$ ethylenediaminetetraacetic acid (EDTA) (Invitrogen) as described elsewhere (Montefiori, 2009). Both cell lines were incubated at $37{ }^{\circ} \mathrm{C}$ in a humidified $5 \% \mathrm{CO}_{2} / 95 \%$ air environment. Unless otherwise specified, all incubations were carried out under these conditions. Viability of the A3R5 used for maintenance and in the assays was acceptable if $>80 \%$. All details regarding the TZM-bl cell cultures and their use in the neutralizing antibody assay were previously reported (Montefiori, 2009; Sarzotti-Kelsoe et al., 2013).

\subsection{Preparation and titration of infectious molecular clones (Env.IMC.LucR)}

The Renilla Luciferase reporter HIV-1 plasmids utilized here were all derived from the previously described NLLucR.T2A-Env.ecto proviral backbone (Edmonds et al., 2010), into which heterologous env gene sequences from different clades were shuttled, including those from transmitted/founder as well as chronic HIV-1 strains. All constructs were isogenic in all HIV open reading frames other than env. Env.IMC.LucR viruses were prepared by transfecting exponentially dividing 293T/17 cells (3-5 × 106 cells in $12 \mathrm{ml}$ DMEM Growth Medium in a T-75 culture flask) with $12 \mu \mathrm{g}$ of IMC plasmid DNA using FuGENE 6 reagent (Promega, USA) and DMEM Growth Medium (Edmonds et al., 2010). The transfection complexes were allowed to incubate for $30 \mathrm{~min}$ at room temperature $\left(18-26{ }^{\circ} \mathrm{C}\right)$. The complexes were then added to the flask of $293 \mathrm{~T} / 17$ cells and incubated for 3-8 h. Following a change of media, the cells were incubated for an additional 24-48 h. The virus-containing media was then harvested from the flasks and the concentration of FBS in the media was adjusted to $20 \%$. The virus-containing media was filtered through a $0.45 \mu \mathrm{m}$ filter, to eliminate cell debris, was aliquoted and frozen at $-80{ }^{\circ} \mathrm{C}$.

For virus titration, Env.IMC.LucR virus stocks were plated in quadruplicate and serially diluted in RPMI Growth Medium. A3R5 cells were added to the plate in RPMI Growth Medium containing an optimized concentration of DiethylaminoethylDextran (DEAE-Dextran, Sigma Chemical Company, St. Louis, $\mathrm{MO})$. DEAE-Dextran is a polycation reagent used to increase 
virus infectivity in the A3R5 assay (McLinden et al., 2013). After a 4-day incubation, $40 \mu$ of supernatant was removed from each well, cells were carefully resuspended, and $75 \mu \mathrm{l}$ of cell suspension was transferred to a solid white plate (Corning Costar). $30 \mu \mathrm{l}$ of Viviren Live Cell Substrate (Promega) was added. After a 4 min incubation at room temperature, measurements of luminescence were detected using a Victor 2 luminometer (Perkin-Elmer Life Sciences, Shelton, CT). The recommended virus dilution to use in the A3R5 assay was calculated to ensure a standardized virus dose of $\sim 50,000-150,000$ relative luminescence unit (RLU) equivalents was used in the assay.

\subsection{Serologic reagents}

All works related to human subjects were in compliance with Institutional Review Board (IRB) protocols approved by the Duke University Health System IRB. Unless otherwise specified, serum samples were collected in serum separator tubes (SST) and plasma samples, in acid citrate dextrose (ACD). The serum sample IBB PT41079 was obtained in the late 1980s from an individual who was infected with subtype B HIV-1 (Interstate Blood Bank) (blood bag collection, preservative addition unknown). Plasma samples were collected from US ("W-series") and African ("10-series") HIV-1-seropositive individuals and provided by Dr. M. Busch, Blood Systems Research Institute (BSRI, San Francisco, CA). Plasma and serum samples were also collected from US and African HIV-1-seropositive and HIV-1-seronegative individuals and provided by the Center for HIV/AIDS Vaccine Immunology (CHAVI). HIVIG-C is a purified IgG antibody from a pool of plasma samples with neutralizing antibody function, derived from HIV-1 subtype C infected individuals. IVIG is a purified IgG from normal HIV-1-seronegative human individuals. $\mathrm{CH} 01$ is a monoclonal antibody (mAb) anti-V1/ V2 conformational epitope of HIV-1 gp120 (Bonsignori et al., 2011, 2012) and VRC-CH31 is a mAb anti-CD4 binding site of HIV-1 (Wu et al., 2011; Bonsignori et al., 2012). Catalent (Madison, WI) manufactured both $\mathrm{CH} 01$ and VRC-CH31 with permission from CHAVI.

\subsection{Neutralizing antibody assay for HIV-1 in A3R5 cells}

Neutralization assays in A3R5 cells were performed by incubating previously titrated Env.IMC.LucR virus with serial 3-fold dilutions of test sample in duplicate in a total volume of $150 \mu \mathrm{l}$ for $45-90 \mathrm{~min}$ in 96 -well flat-bottom culture plates. Exponentially growing cells (90,000 cells, viability $>80 \%$, calculated using $0.4 \%$ trypan blue solution with a manual or automated cell counter) in $100 \mu$ of RPMI Growth Medium containing $25 \mu \mathrm{g} / \mathrm{ml}$ DEAE-Dextran were added to each well. One set of control wells received cells + virus (virus control) and another set received cells only (background control). The plate was incubated for 4 days. After the incubation, $90 \mu \mathrm{l}$ of supernatant was removed from each well, cells were carefully resuspended, and $75 \mu \mathrm{l}$ of cell suspension was transferred to a solid white plate (Corning Costar). $30 \mu \mathrm{l}$ of a Renilla Luciferase reporter gene assay system reagent (Viviren Live Cell Substrate, Promega) was added. After a 4 min incubation at room temperature, measurements of luminescence were detected using a Victor 2 luminometer (Perkin-Elmer Life
Sciences, Shelton, CT). The luminometer was calibrated annually by the manufacturer and its performance was validated by the laboratory monthly using a Luminometer Reference Microplate (Harta Instruments), as previously described (Ozaki et al., 2012). Neutralization titers [ $50 \%$ inhibitory dose (ID50) or the $50 \%$ inhibitory concentration (IC50)] were defined as the reciprocal of the serologic reagent dilution (or concentration for purified reagents) that caused a 50\% reduction in RLUs compared to virus control wells after subtraction of background RLUs. Pass/fail quality control (QC) criteria for the assay were as follows: 1) The average RLUs of virus control wells are $>10$ times the average RLUs of cell control wells; 2) The \% coefficient of variation (CV) of RLUs in the virus control wells is $\leq 30 \%$; 3 ) The \% CV for duplicate wells is $\leq 30 \%$ for sample dilutions that yield at least $40 \%$ neutralization; 4) The neutralization curves are smooth and linear around the 50\% neutralization cut-off; and 5) Positive control titers must be within 3-fold of the mean (from LeveyJennings plots) of the previous values for that particular control-virus combination. Data that failed the QC criteria were excluded from the analysis.

\subsection{Flow cytometry}

The expression of CD4, CCR5 and CXCR4 on A3R5 cells was monitored over time by flow cytometry. Cells were stained for CD4, CCR5 and CXCR4 surface expression using the following mouse anti-human mAbs: CD4-PE (Cat. No. 555347), CD195-PE (Cat. No. 556042) and CD184-PE (Cat. No. 555974), respectively. Mouse $\operatorname{IgG}_{1}$, $\kappa$ PE (Cat. No. 556650) was used for background staining in the case of CD4. Rat IgG2a, $\kappa$ PE (Cat. No. 559317) was used for background staining of CCR5 and CXCR4. All antibodies were purchased from BD/Pharmingen. Approximately 10,000 events were acquired using a FACSCalibur flow cytometer.

\subsection{Statistical analysis}

All IC50/ID50 values were calculated using a formally validated Excel-based macro or web-based neutralizing antibody (Nab) tool (Piehler et al., 2011), both of which utilize average virus and cell control RLU values as well as duplicate test well RLU values to calculate the neutralizing antibody titer as a function of the reduction of luciferase reporter gene expression. Statistical analyses were performed using SAS Version 9.2. Boundaries for comparisons were typically made using a 3-fold margin and the error rate was tested using a binomial test. The accuracy assessment was based on a non-inferiority test with the TZM-bl assay as the standard reference assay. This assessment was made via regression models using PROC MIXED with random effects for the samples.

\section{Results}

\subsection{Optimization of the A3R5 assay}

\subsubsection{Optimal A3R5 cell density for the assay}

Experiments were designed to identify the input number of A3R5 cells/well for optimal sensitivity of the neutralization assay. Four Env.IMC.LucR viruses (two clade B and two clade $C$ viruses) were assayed against three plasma samples and two mAbs at increasing A3R5 input cell densities $(22,500$, 

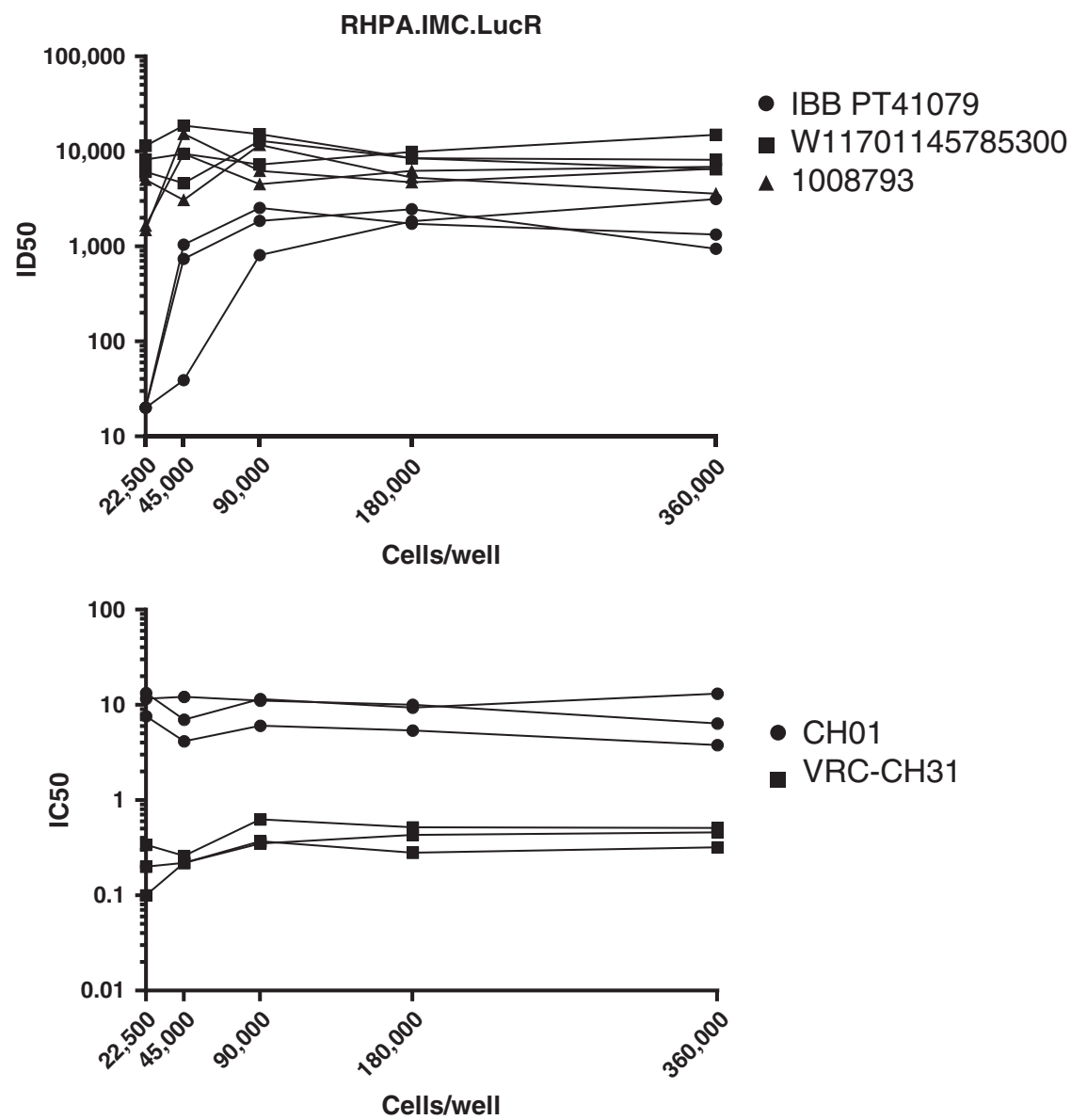

Fig. 1. Optimal A3R5 cell number for assay sensitivity. RHPA.IMC.LucR virus was assayed against three plasma samples (upper panel) and two mAbs (lower panel) in three repeats at increasing A3R5 input cell densities (22,500, 45,000, 90,000, 180,000, 360,000 cells/well). Luminescence was measured in a 4-day assay. Results are expressed as ID50 (upper panel) or IC50 (lower panel).

45,000, 90,000, 180,000, and 360,000 cells/well). Separate assay plates were used for each cell density. Representative results obtained with RHPA.IMC.LucR virus (Fig. 1) demonstrate that ID50/IC50 values reached a plateau at a cell density of 90,000 cells/well, while more variability between reagents and within repeats was observed at lower cell densities. Cell densities higher than 90,000 cells/well induced a loss in neutralization sensitivity with some viruses. Thus, a cell density of 90,000 cells/well was chosen as the optimal input cell number for the neutralization assay. This input cell number ( 90,000 cells/ well) also permitted optimal measurements of infection in virus infectivity assays (data not shown).

\subsubsection{Kinetics of infection of A3R5 cells by Env.IMC.LucR viruses}

A3R5 cells are susceptible to a readily detectable level of infection by full-length HIV-1 molecular clones (Env.IMC.LucR) but not by most Env-pseudotyped viruses. We examined the kinetics of Env.IMC.LucR detection in A3R5 cells. Serially diluted Env.IMC.LucR viruses in quadruplicate wells were used to infect A3R5 cells. Luminescence was read at 2, 4, 7 and 9 days post-infection (Fig. 2). RLU values increased with time in culture. However, a prozone effect at the highest virus concentrations, caused by virus-induced syncytium formation and consequent cell toxicity, was observed at 7 and 9 days post-infection. In order to avoid cell toxicity, the optimal time for viral detection was judged to be 4 days.

\subsubsection{Optimal viral dose for maximum assay sensitivity}

We next determined the optimal virus inoculum dose for best neutralization sensitivities. Serologic reagents were serially diluted and tested against Env.IMC.LucR viruses at the optimal RLU equivalents dose selected in the infectivity assay $(1 \times)$, as well as at $1 / 3 \times, 1 / 10 \times, 3 \times$, and $10 \times$ the optimal RLU equivalents' dose (Fig. 3 ). The neutralization curves generated by all serologic reagents tested showed a consistent pattern of linearity over an approximate range of $20-80 \%$ reduction in RLU (\% neutralization). The $50 \%$ reduction in RLU was chosen to determine neutralization results (IC50/ID50), as the value lies midway in the linear portion of the curves. Values in this range are directly proportional to the concentration of neutralizing antibodies in the sample. Additionally, these results demonstrated neutralizing responses by all reagents tested, regardless of input viral dose. In fact, the IC50/ID50 values for all reagents differed by $<3$ fold over a 100 -fold range of virus input doses, 

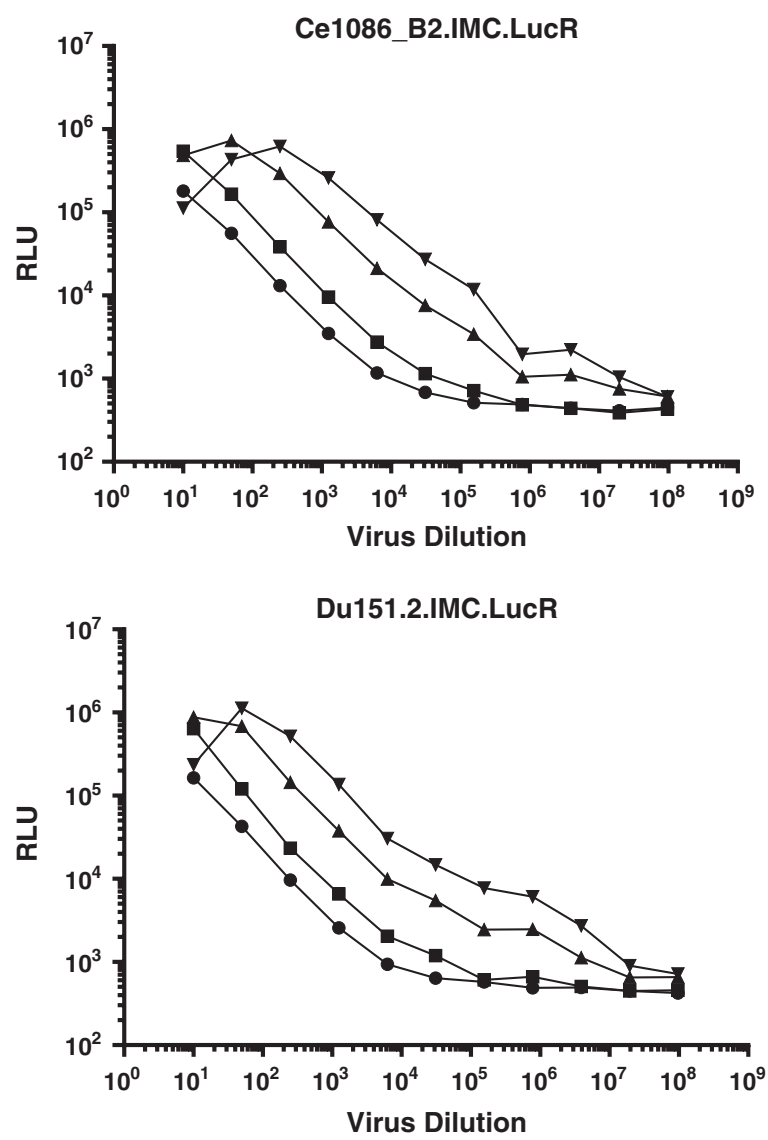

\section{Incubation Time}

- 2 days

- 4 days

- 7 days

$\checkmark 9$ days

Fig. 2. Kinetics of infection of A3R5 cells by Env.IMC.LucR viruses. Serially diluted Ce1086_B2.IMC.LucR and DU151.2.IMC.LucR viruses in quadruplicate wells were used to infect A3R5 cells (90,000 cells/well) in four replicate plates, one for each of the time points studied. Luminescence was read at 2 days, 4 days, 7 days and 9 days post-infection. Results are expressed as RLU equivalents for each virus dilution used.

indicating that minor differences in the virus dose used in the A3R5 assay are unlikely to affect the neutralization results. In order to optimize neutralization sensitivity and minimize cell toxicity, the recommended virus dilution to use in the assay was selected to be $\sim 50,000-150,000$ RLU equivalents.

\subsubsection{Non-specific background neutralizing activity of plasma and sera}

For an assay to be specific, it is important to establish a negativity threshold. Since both serum and plasma samples may be tested for neutralization in immune monitoring studies, a set of twenty serum and twenty plasma samples from HIV-negative individuals (as defined by $<48$ DNA copies/ml) was tested against ten different Env.IMC.LucR viruses, five clade $B$ and five clade $C$ (data not shown). A higher background was observed for all plasma samples, compared to sera. A non-parametric calculation was performed on each of the 10 Env.IMC.LucR viruses individually. An ID50 $=23.5$ for serum samples and an ID50 $=82.5$ for plasma samples were established as the negativity thresholds or Limit of Blank (LOB) values for all viruses tested. The LOB corresponds to the Limit of Detection (LOD) for this assay. Conversely, when a parametric interval [mean titer value of negative samples $+1.96(\mathrm{SD})=$ Limit of Blank (LOB)] was applied across all viruses, the LOB was ID50 $=21$ for serum and 52.6 for plasma samples. Based on both statistical analyses and scientific judgment, the final LOB was determined to be $\mathrm{ID} 50=20$ for serum samples and $\mathrm{ID} 50=60$ for plasma samples. The false positive rate across all viruses, using these LOB/LOD values, was 2.5\% with an exact binomial 95\% Confidence Interval $(\mathrm{CI})$ of $(0.8,5.7)$. These acceptance criteria were applied to the analysis of the subsequent validation results, derived from testing a completely new set of HIV-negative serum and plasma samples.

\subsection{Validation of the A3R5 assay}

The assay conditions and acceptance criteria defined in the optimization studies and described in Standard Operating Procedures (SOPs) containing pass/fail QC criteria, were utilized in the assay validation testing described in the following paragraphs. In GCLP compliance, a prospective validation plan with pre-set acceptance criteria, based on the optimization results, was composed and implemented. Key assay parameters included specificity, accuracy, precision, limit of detection and quantitation, specificity, linearity and range, and robustness. Results of the validation experiments were statistically analyzed 


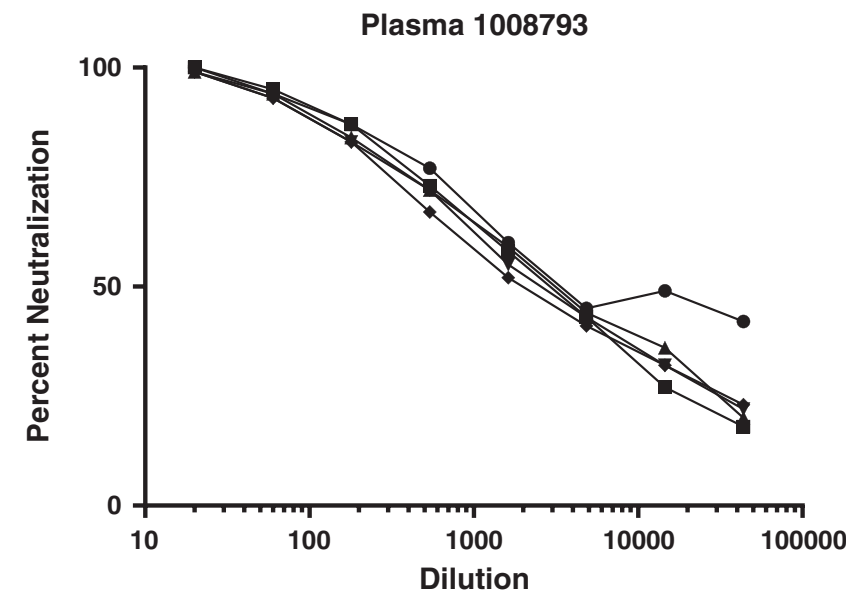

Virus Dose

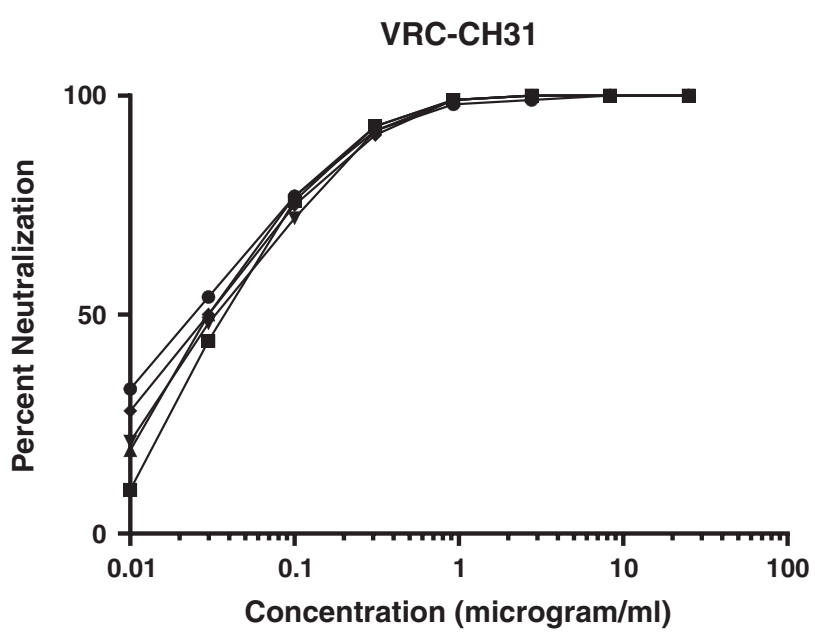

- $1 / 10 x$

- $1 / 3 x$

$\Delta 1 \mathrm{x}$

$\checkmark 3 x$

- $10 x$

Concentration (microgram/ml)

Fig. 3. Optimal viral dose for maximum assay sensitivity. Plasma sample (1008793) and mAb VRC-CH31 tested against SC22.3C2.IMC.LucR virus at $1 / 3 \times, 1 / 10 \times$, $3 \times$, and $10 \times$ the optimal ( $1 \times)$ RLU equivalent doses. Results are expressed as \% neutralization at each reagent dilution (upper panel) or concentration (lower panel).

and used to generate a final validation document (see schematic, Todd et al., 2013).

\subsubsection{Specificity}

The specificity of an analytical procedure expresses the ability to unequivocally assess the analyte in the presence of other components, which may be expected to be present. In order to test for non-specific background activity of normal human sera and plasma samples (negativity threshold), forty human sera and forty human plasma samples from HIV-1 seronegative individuals were tested against a standard panel of seven Env.IMC.LucR viruses (total: 280 sera/virus combinations and 280 plasma/virus combinations). As observed in the optimization experiments, the background activity of plasma samples was always higher than that of serum samples. When serum samples were analyzed ( 279 serum/virus combinations, one sample failed the assay QC), 274 had ID50 results $\leq 20$, for a false positive rate across all viruses $=1.79 \%$, which is within the acceptance criteria of $2.5 \%$ (see Section 3.1.4) (Fig. 4, Table 1). The analysis of the results obtained individually for each of the seven Env.IMC.LucR viruses tested, indicated that four serum samples, tested against Du151.2.IMC.LucR virus, exceeded the LOD (ID50: $>20$, false positive rate $>2.5 \%$ ). When these four samples were re-tested against the same batch, as well as two additional batches of Du151.2.IMC.LucR, ID50 titers $>20$ were not detected (data not shown), indicating that their responses were truly false positive. The analysis of plasma samples (278 plasma/virus combinations, two samples failed the assay QC), indicated that 275 had ID50 results $\leq 60$, for an acceptable false positive rate across all viruses $=1.09 \%$ (Fig. 4 , Table 1). Therefore, the LOD pre-selected for the A3R5 assay in the optimization studies was validated by these results.

DEAE-Dextran is a polycation reagent used to increase virus infectivity (McLinden et al., 2013; Sarzotti-Kelsoe et al., 2013). We determined the potential interference effect of DEAE-Dextran on the neutralization assay by testing three neutralizing reagents, in three repeats, against three Env.IMC.LucR viruses in the presence and absence of DEAE-Dextran in the assay. Table 2 shows that the mean titers obtained in the presence or absence of DEAE-Dextran were not statistically different $(p=0.75)$, and were all within the 3 -fold boundary across all viruses, indicating that the inclusion of DEAE-Dextran in the assay does not significantly affect the neutralization titers.

Heat-inactivation of serum and plasma samples is normally used to disarm non-specific blood factors, such as complement, 


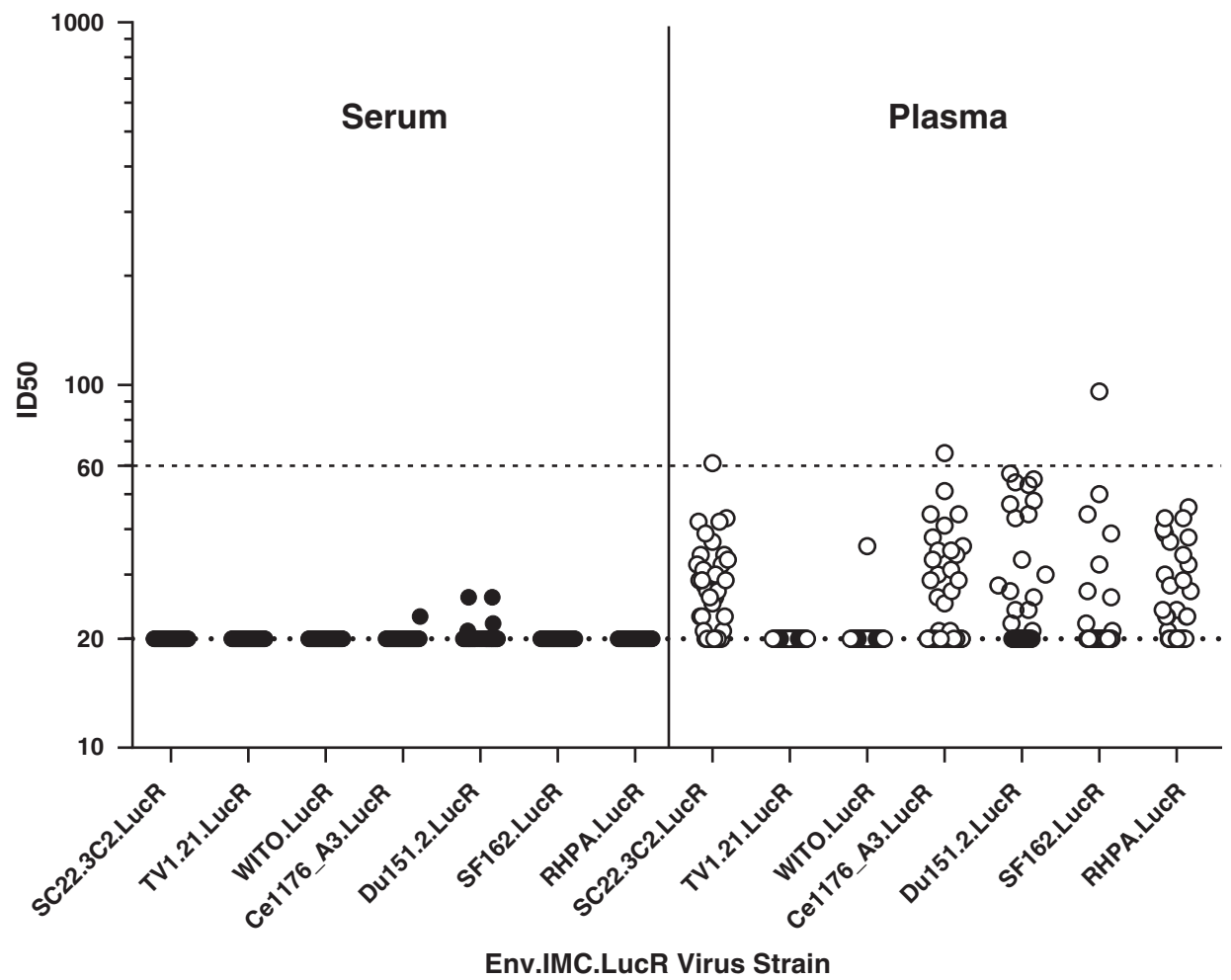

Fig. 4. Non-specific background activity of HIV-negative serum and plasma samples. Forty human sera and forty human plasma samples from HIV-negative individuals (<48 HIV-1 DNA copies/ml, kindly provided by CHAVI) were tested against seven Env.IMC.LucR viruses, in a standard A3R5 4-day assay. All samples were heat-inactivated at $56{ }^{\circ} \mathrm{C}$ for $1 \mathrm{~h}$ before testing. Results are expressed as ID50 titers. Horizontal dotted lines represent the LOB (ID50 $=20$ for sera; ID50 $=60$ for plasma).

which might otherwise interfere with virus neutralization in the A3R5 assay. We tested whether heat-inactivation of serum and plasma samples at $56{ }^{\circ} \mathrm{C}$ for $1 \mathrm{~h}$ affected the ability of twenty plasma and twenty sera from HIV seropositive samples to neutralize three Env.IMC.LucR viruses (Table 3). We tested 114 serologic reagent/virus combinations and there were three values (in response to WITO.IMC.LucR) outside the 3-fold boundary (2.63\%), which is within the acceptable $5 \%$ error rate. Overall, Table 3 demonstrates that the ID50 comparison titers for neutralizing reagents (plasma and sera) tested before and after heat-inactivation were within the preset acceptance

Table 1

Non-specific background of HIV-1 seronegative plasma and sera.

\begin{tabular}{llll}
\hline Virus & Sample type & N & False positives \\
\hline Ce1176_A3.IMCLucR & Plasma & 40 & 1 \\
& Serum & 40 & 1 \\
Du151.2.IMC.LucR & Plasma & 40 & 0 \\
& Serum & 40 & 4 \\
RHPA.IMC.LucR & Plasma & 40 & 0 \\
SC22.3C2.IMC.LucR & Serum & 40 & 0 \\
& Plasma & 40 & 1 \\
SF162.IMC.LucR & Serum & 40 & 0 \\
TV1.21.IMC.LucR & Plasma & 38 & 1 \\
& Serum & 40 & 0 \\
WITO.IMC.LucR & Plasma & 40 & 0 \\
& Serum & 39 & 0 \\
& Plasma & 40 & 0 \\
& Serum & 40 & 0 \\
\hline
\end{tabular}

criteria (within 3-fold of the mean titer with an error rate of $5 \%)$ across viruses.

\subsubsection{Precision}

Precision is a measurement of the scatter or random error between repeated measurements. Precision was considered at three levels: repeatability, intermediate precision, and reproducibility.

To test the repeatability (intra-operator and intra-assay variability) of the assay, three serologic reagents were tested against three Env.IMC.LucR viruses (Fig. 5). A total of twenty replicates of each sample, tested in duplicate wells by one operator were assayed in different positions on different plates in one setting. All replicates of each sample with low, medium, and high neutralization titers were within 3-fold of the mean.

For intermediate precision testing (intra-laboratory variability), samples included four serologic reagents and four Env.IMC.LucR viruses. The samples were blinded to the operators. Two different operators in the same laboratory performed these assays on three different days, using three different luminometers. The readings from the three different luminometers were examined from the same plate. The pre-set acceptance criteria required that the IC50 and ID50 titers should be within 3 -fold of the mean with an error rate of $10 \%$. The results in Table 4 show that $93.6 \%$ of the serologic reagent/virus combination titers were within 3-fold of the mean, with an acceptable error rate of $6.4 \%$. The intermediate precision data 
Table 2

Effect of DEAE-Dextran on neutralizing antibody responses.

\begin{tabular}{|c|c|c|c|c|c|}
\hline Virus & Sample & DEAE-Dextran (ID50) & No DEAE-Dextran (ID50) & 3-Fold boundary & Outside boundary $^{*}$ \\
\hline \multirow[t]{3}{*}{ TV1.21.IMC.LucR } & $\mathrm{CH} 01$ & 328 & 548 & $(984,109)$ & 0 \\
\hline & VRC-CH31 & 20 & 20 & $(60,7)$ & 0 \\
\hline & W11701145785300 plasma & 1315 & 1229 & $(3945,438)$ & 0 \\
\hline \multirow[t]{3}{*}{ WITO.IMC.LucR } & $\mathrm{CH} 01$ & 8651 & 10,367 & $(25,953,2884)$ & 0 \\
\hline & VRC-CH31 & 3530 & 3817 & $(10,590,1177)$ & 0 \\
\hline & W11701145785300 plasma & 1077 & 981 & $(3231,359)$ & 0 \\
\hline \multirow[t]{3}{*}{ Ce1176_A3.IMC.LucR } & $\mathrm{CH} 01$ & 9854 & 7248 & $(29,562,3285)$ & 0 \\
\hline & VRC-CH31 & 669 & 613 & $(2007,223)$ & 0 \\
\hline & W11701145785300 plasma & 210 & 362 & $(630,70)$ & 0 \\
\hline
\end{tabular}

* All p-values $=0.75$.

Table 3

Effect of heat-inactivation on neutralizing antibody responses.

\begin{tabular}{llccc}
\hline Virus & Sample & Heat-inactivation $(\mathrm{N})$ & No heat-inactivation (N) \\
\hline WITO.IMC.LucR & Plasma & 20 & 20 & 16 \\
& Sera & 16 & 20 & 2 \\
TV1.21.IMC.LucR & Plasma & 20 & 20 & 0 \\
& Sera & 20 & 19 & 19 \\
RHPA.IMC.LucR & Plasma & 19 & 114 & 0 \\
Total & Sera & 19 & $3^{*}$
\end{tabular}

${ }^{*}$ p-Value $=0.83$.

Table 4

Intermediate precision assessment.

\begin{tabular}{|c|c|c|c|c|c|c|}
\hline Virus & Oper. & Sample & $\mathrm{N}$ & Mean & 3-Fold boundary & Outside of boundary \\
\hline \multirow[t]{8}{*}{ Ce1176_A3.IMC.LucR } & 1 & VRC-CH31 & 27 & 467.4 & $(155.8,1402.3)$ & 0 \\
\hline & & IBB & 26 & 20.0 & $(6.7,60.0)$ & 0 \\
\hline & & IVIG & 8 & 20.0 & $(6.7,60.0)$ & 0 \\
\hline & & W04 & 24 & 29.6 & $(9.9,88.9)$ & 0 \\
\hline & 2 & VRC-CH31 & 27 & 826.6 & $(275.5,2479.7)$ & 0 \\
\hline & & IBB & 27 & 20.0 & $(6.7,60.0)$ & 0 \\
\hline & & IVIG & 9 & 20.0 & $(6.7,60.0)$ & 0 \\
\hline & & W04 & 27 & 22.9 & $(7.6,68.7)$ & 0 \\
\hline \multirow[t]{8}{*}{ DU151.2.IMC.LucR } & 1 & VRC-CH31 & 27 & $14,872.3$ & $(4957,44,617)$ & 0 \\
\hline & & IBB & 27 & 20.0 & $(6.7,60.0)$ & 0 \\
\hline & & IVIG & 9 & 20.0 & $(6.7,60.0)$ & 0 \\
\hline & & W04 & 27 & 60.0 & $(20.0,179.9)$ & 0 \\
\hline & 2 & VRC-CH31 & 27 & $16,804.1$ & $(5601,50,412)$ & 0 \\
\hline & & IBB & 27 & 20.4 & $(6.8,61.1)$ & 0 \\
\hline & & IVIG & 9 & 20.0 & $(6.7,60.0)$ & 0 \\
\hline & & W04 & 27 & 35.3 & $(11.8,106.0)$ & 0 \\
\hline \multirow[t]{8}{*}{ SC22.3C2.IMC.LucR } & 1 & VRC-CH31 & 27 & $25,125.4$ & $(8375,75,376)$ & 1 \\
\hline & & IBB & 27 & 112.4 & $(37.5,337.2)$ & 15 \\
\hline & & IVIG & 9 & 20.0 & $(6.7,60.0)$ & 0 \\
\hline & & W04 & 27 & 2819.7 & $(939.9,8459.1)$ & 8 \\
\hline & 2 & VRC-CH31 & 27 & $30,694.6$ & $(10,232,92,084)$ & 1 \\
\hline & & IBB & 27 & 146.1 & $(48.7,438.3)$ & 9 \\
\hline & & IVIG & 9 & 20.0 & $(6.7,60.0)$ & 0 \\
\hline & & W04 & 27 & 2088.9 & $(696.3,6266.7)$ & 9 \\
\hline \multirow[t]{8}{*}{ TV1.21.IMC.LucR } & 1 & VRC-CH31 & 27 & 20.0 & $(6.7,60.0)$ & 0 \\
\hline & & IBB & 27 & 43.2 & $(14.4,129.6)$ & 0 \\
\hline & & IVIG & 9 & 20.0 & $(6.7,60.0)$ & 0 \\
\hline & & W04 & 27 & 174.6 & $(58.2,523.8)$ & 0 \\
\hline & 2 & VRC-CH31 & 27 & 20.0 & $(6.7,60.0)$ & 0 \\
\hline & & IBB & 27 & 61.9 & $(20.6,185.6)$ & 0 \\
\hline & & IVIG & 9 & 20.0 & $(6.7,60.0)$ & 0 \\
\hline & & W04 & 27 & 196.0 & $(65.3,588.1)$ & 3 \\
\hline Total & & & 715 & & & $46^{*}$ \\
\hline
\end{tabular}

${ }^{*}$ p-Value $=0.9$. 

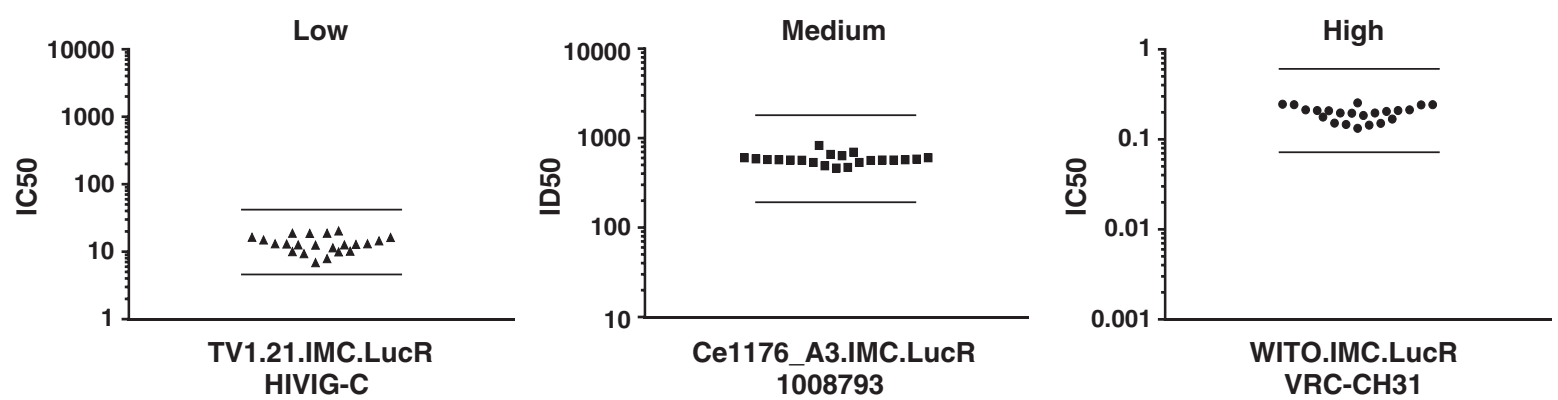

Fig. 5. Intra-assay and intra-operator variability. HIVIG-C, plasma sample 1008793, and VRC-CH31 were tested against three Env.IMC.LucR viruses, in twenty replicates of each sample, tested in duplicate wells by one operator, in different positions on different plates in one assay setting. Results are expressed as IC50/ID50 titers for each of the serologic reagent/virus combination. Horizontal bars represent the 3-fold boundaries.

were also analyzed in a regression model using the natural $\log$ of the titer values to compare the differences between operators within specific virus/sample combinations. The overall operator effect in the regression model was not significant $(\mathrm{p}=0.197)$. However, two samples (W04 and IBB) against SC22.3C2.IMC.LucR demonstrated significant differences at the alpha 0.01 level. Samples were not available for repeat testing in this case.
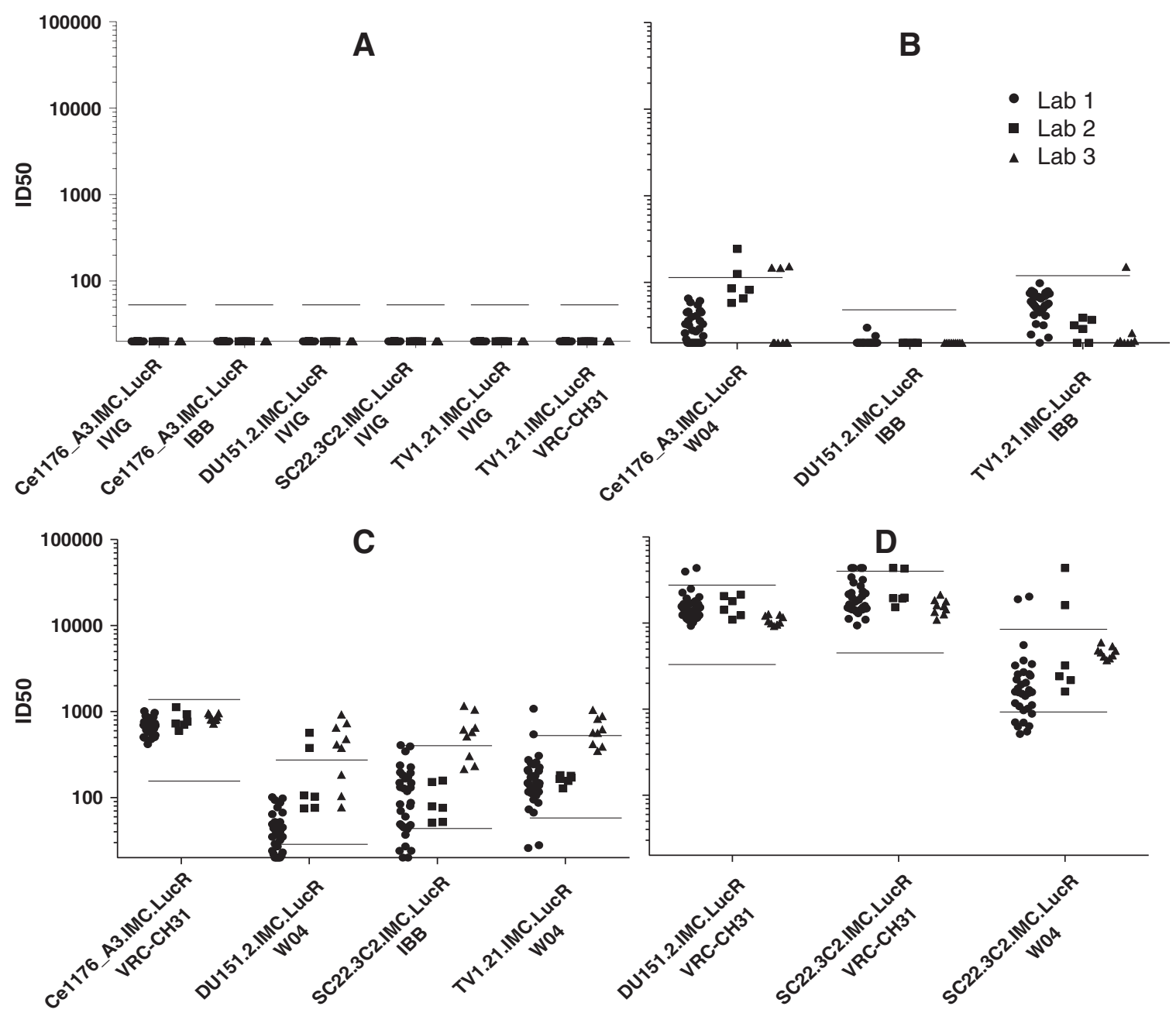

Fig. 6. Reproducibility of the A3R5 assay in domestic and international laboratories. ID50 titers from three participating laboratories testing serologic reagents with negative (ID50 < 20, panel A), low (ID50 21-100, panel B), medium (ID50 101-1000, panel C), and high (ID50 > 1000, panel D) neutralization responses. The different symbols represent the results from operators within each of the three participating laboratories. Bars indicate the upper and lower limits of the acceptance ranges. The $\mathrm{x}$-axes list the various virus/serologic reagent combinations used in the tests. 
Finally, reproducibility (inter-laboratory variability) was evaluated by testing a panel of known HIV seropositive reagents for neutralization of four Env.IMC.LucR viruses (Fig. 6). One international and two domestic laboratories tested the same panel of blinded serologic reagents against the same set of Env.IMC.LucR viruses provided in the kit, using their own A3R5 cell stocks. These three laboratories successfully participate in the proficiency testing program for neutralizing antibody assay in TZM-bl assay (Todd et al., 2012). Items included in the kits provided to the three laboratories were: the same blinded serologic reagents with negative (ID50 $<20$, panel A), low (ID50 21-100, panel B), medium (ID50 101-1000, panel C), and high (ID50 > 1000, panel D) neutralization titers; four open-labeled Env.IMC.LucR viruses; the same SOP. Three of the blinded serologic reagents were provided in triplicate, to analyze the intra-operator variability within testing laboratories. A minimum of two operators/laboratory performed the test, for a total of 16 operators tested. Due to the limited number (three) of laboratories conducting the A3R5 assay at the time of the validation studies, these reproducibility results were evaluated using the acceptance criteria established for intermediate precision, as this was the first inter-laboratory comparison performed for the A3R5 assay. All laboratories accurately identified the serologic reagents in each of the four categories (negative, low, medium, and high titers) (Fig. 6). Within each category, the results were as follows: all negative titer values (100\%) were within 3-fold of the mean value (Fig. 6, A), indicating complete congruency; $98 \%$ of the low titer values were within 3 -fold of the mean value (Fig. 6, B), indicating a $2 \%$ error rate; $72 \%$ of the medium titer values were within 3 -fold of the mean value (Fig. 6, C), indicating a $28 \%$ error rate; $89 \%$ of the high titer values were within 3-fold of the mean value (Fig. 6, D), indicating an error rate of $11 \%$. The error rate was higher in the medium and high neutralization titer categories ( $C$ and $D$ ) for IBB and W04 reagents. All tests performed with the VRC-CH31 $\mathrm{mAbs}$ showed complete reproducibility between all operators. Overall, $89.1 \%$ of all serologic reagent/virus combination titers were within 3-fold of the mean, indicating an overall error rate of $11 \%$, which is outside the acceptance criteria. Finally, the intra-operator variability was analyzed using the $\log 10$ ID50 values of the three reagents' triplicate repeats (Fig. 7) used in the reproducibility tests. The graphical display of the $95 \%$ CIs for each operator in rank order indicates random intra-operator variability between the three testing laboratories.

\subsubsection{Accuracy}

The accuracy of an analytical procedure expresses the closeness of agreement between a conventional true value, or an accepted reference value, and the value found. Although a true "gold standard" for the HIV-1 neutralizing antibody assay does not exist, we performed a comparison test of the A3R5 assay with the validated TZM-bl assay (Sarzotti-Kelsoe et al., 2013). Ten plasma and ten serum samples from HIV-1-positive individuals were tested for neutralization against Env.IMC.LucR viruses using both the TZM-bl assay and the A3R5 assay. The assumption was made that the ability of the A3R5 assay at detecting neutralization of Env.IMC.LucR viruses was as accurate as, or non-inferior to, the TZM-bl assay. We used the log10 transformed ID50 titer values as the outcome. A paired t-test was performed using SAS Proc Mixed with random effects for sample to test the null hypothesis that the mean A3R5 titers are less than or equal to $90 \%$ of the TZM-bl titers (Ho: $\mu \mathrm{A} \leq \mu \mathrm{T} * 0.9$ ). The alternative hypothesis is that the mean A3R5 IC50/ID50 is greater than $90 \%$ of the TZM-bl ID50 (Ha: $\mu \mathrm{A}>\mu \mathrm{T} * 0.9$ ). If the null hypothesis were rejected, we would accept the alternative hypothesis that the A3R5 assay is at least as accurate/non-inferior to the TZM-bl assay, with a $10 \%$ non-inferiority margin. Table 5 shows the results (all p-values $<0.001$ ), indicating that the A3R5 assay is as accurate/non-inferior to the TZM-bl assay at detecting positive neutralization of Env.IMC.LucR viruses, with a $10 \%$ non-inferiority margin.

Furthermore, ID50 titers were plotted as coordinates (x-axis = TZM-bl values, $\mathrm{y}$-axis $=$ A3R5 values, Fig. 8$)$ to graphically show the comparison between A3R5 and TZM-bl titers obtained for the same serologic reagents. The diagonal line depicts $\mathrm{x}=\mathrm{y}$ for the TZM-bl ID50 values = A3R5 ID50 values. A3R5 titers higher than the TZM-bl titers would be plotted above the $\mathrm{x}=\mathrm{y}$ line, while equivalency with the TZM-bl values would show the A3R5 titer values on the $\mathrm{x}=\mathrm{y}$ line $( \pm 10 \%$ error rate). Fig. 8 demonstrates that for 83\% of the Env.IMC.LucR viruses, the A3R5 titers were higher than TZM-bl titers, while SF162.LucR (a tier 1, easy to neutralize virus) was neutralized similarly in both assays. This outcome agrees with the known increased sensitivity of the A3R5 assay with tier 2 viruses (Montefiori et al., 2012; McLinden et al., 2013; DeCamp et al., 2014).

\subsubsection{Linearity, limit of quantitation (LOQ), and range}

Linearity is the ability of an analytical procedure to obtain test results within a given range that are directly proportional to the concentration of analyte in the sample. The range of an assay is the interval between the upper and lower concentration of an analyte in the sample for which it has been demonstrated that the analytical procedure has a suitable level of precision, accuracy, and linearity. Neutralization curves generated with serologic reagents show a consistent pattern of linearity over an

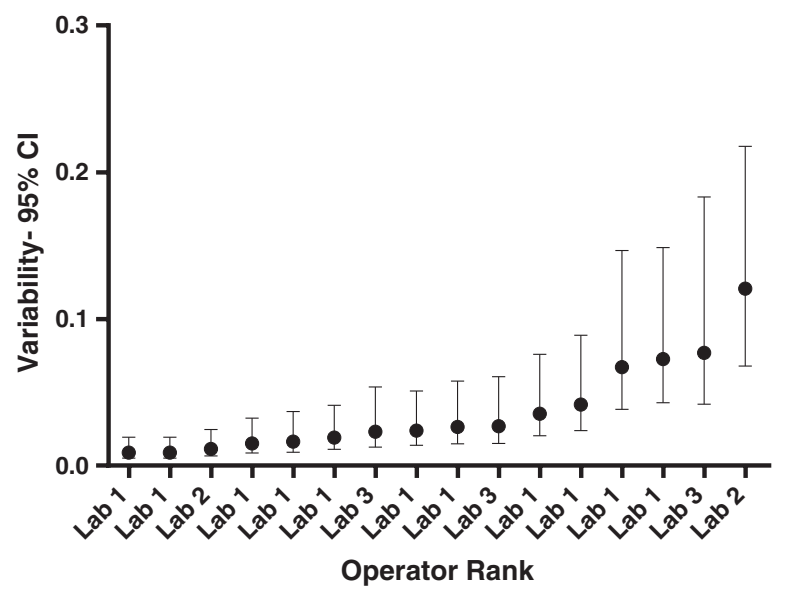

Fig. 7. Repeatability within reproducibility. Graphical display of the $95 \%$ confidence intervals (CIs) of the $\log 10$ ID50 values obtained for three reagents' triplicate repeats by each laboratory operator (identified by Labs 1-3 only) in rank order. 
Table 5

Accuracy of A3R5 titers compared to TZM-bl titers.

\begin{tabular}{llllll}
\hline Virus & A3R5 Avg. titer & TZM-bl Avg. titer & Avg. difference & Difference to rule out & Difference boundary \\
\hline Ce0393_C3.IMC.LucR & 4.35 & 1.82 & 2.54 & -0.18 & $(2.24,2.84)$ \\
9020.A13.IMC.LucR & 4.41 & 1.82 & 2.59 & -0.18 & $(2.29,2.89)$ \\
SF162.IMC.LucR & 3.87 & 3.76 & 0.11 & -0.38 & $(-0.05,0.27)$ \\
RHPA.IMC.LucR & 4.01 & 1.92 & 2.09 & -0.19 & $(1.88,2.30)$ \\
WITO.IMC.LucR & 3.28 & 1.69 & 1.59 & -0.17 & $(1.10,2.09)$ \\
TV1.21.IMC.LucR & 3.55 & 2.79 & 0.77 & -0.28 & $(0.65,0.88)$ \\
\hline
\end{tabular}

$*$ All p-values $<0.001$.

approximate range of $20-80 \%$ reductions in RLU (Fig. 3). Values in this range are directly proportional to the concentration of neutralizing antibodies in the sample.

LOQ is the lowest concentration of analyte that can be quantified with acceptable precision. The LOQ of the A3R5 assay was determined by the highest dilution of serum/ plasma or lowest concentration of purified antibody that reduces RLU by $50 \%$ relative to the virus control wells. This cut-off was chosen because it lies midway in the linear portion of neutralization curves (20-80\% reductions in RLU) and is above the level of background noise in the assay. Failure to score at least 50\% reduction of RLU at any sample dilution constitutes a negative test.

\subsubsection{Robustness}

Robustness is a measure of the capacity of an analytical procedure to remain unaffected by small, but deliberate variations in method parameters, providing an indication of its reliability during normal usage. Extensive robustness experiments were conducted during the optimization/ qualification phase of the assay.

We determined the stability of the cell line over time in culture for receptor and co-receptor expression (McLinden et al., 2013). Variation in the fraction of cells expressing HIV-1 fusion receptors (CD4 and either CCR5 or CXCR4) may affect the performance of the assay by altering the magnitude and kinetics of infection. The expression of CD4, CCR5, and CXCR4 by cultured A3R5 cells was monitored over time by flow cytometry. As shown in Table 6, the three fusion receptors were stably expressed on A3R5 cells for at least 12 weeks in culture. A decrease in CCR5 expression occurred by week 9 in one evaluation, although it did not affect infectivity or neutralization results performed in parallel (data not shown). Therefore, A3R5 cells should be maintained in culture for $\leq 3$ months.

Further robustness studies defined the parameters most affected by laboratory working conditions. The reliability of the A3R5 assay results is dependent upon the number of A3R5 cells/well (90,000 cells/well) (Fig. 1), the incubation time for the assay (92-100 h), the stability of reagents upon multiple freeze and thaw cycles (three freeze/thaw cycles at $-80{ }^{\circ} \mathrm{C}$, or storage at $4{ }^{\circ} \mathrm{C}$ for a maximum of five months), and the time for luminescence reading $(0.5-1 \mathrm{~s})$ (data not shown).

\section{Discussion}

The results of the A3R5 assay validation demonstrated specificity, accuracy, precision, linearity, robustness, and limits of detection and quantitation.
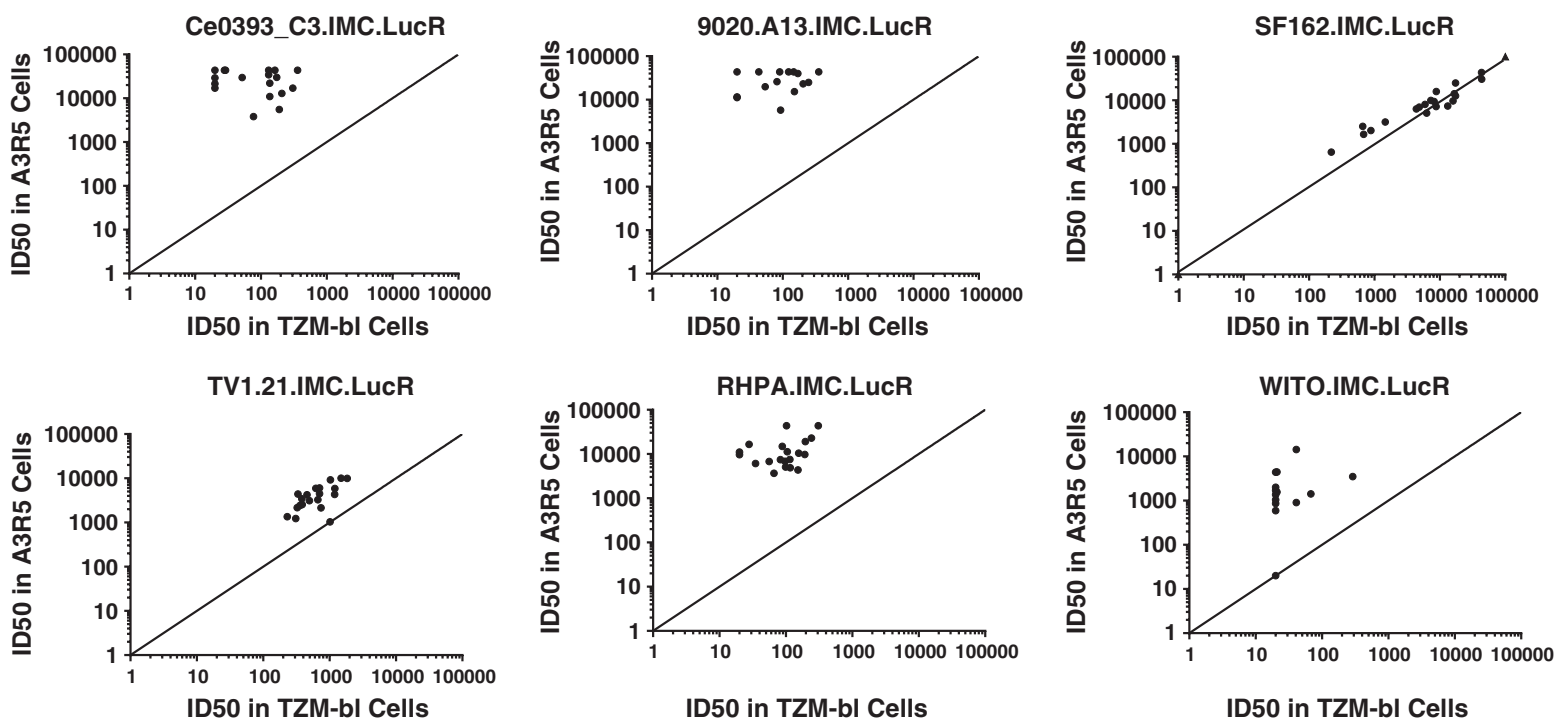

Fig. 8. Comparison of the A3R5 and the TZM-bl neutralization assays for the same set of serologic reagents. Dots represent ten HIV-positive plasma and ten serum samples tested for neutralization against six Env.IMC.LucR viruses in the TZM-bl assay and the A3R5 assay. The diagonal line depicts $\mathrm{x}=\mathrm{y}$ (TZM-bl ID50 = A3R5 ID50 values). 
Table 6

Flow cytometric analysis of CD4, CCR5 and CXCR4 cell surface expression on A3R5 cells.

\begin{tabular}{lccc}
\hline $\begin{array}{l}\text { Weeks in } \\
\text { culture }\end{array}$ & $\begin{array}{l}\text { \% CD4 } \\
\text { positive }^{\mathrm{a}}\end{array}$ & $\begin{array}{l}\text { \% CCR5 } \\
\text { positive }^{\mathrm{a}}\end{array}$ & $\begin{array}{c}\text { \% CXCR4 } \\
\text { positive }^{\mathrm{a}}\end{array}$ \\
\hline 3 & 86.7 & 80.7 & 99.8 \\
$6-7$ & 86.4 & 76.6 & 99.8 \\
9 & 77.4 & 58.4 & 99.7 \\
12 & 81.8 & 57.8 & 99.9 \\
$15-17$ & 72.2 & 62.1 & 99.5 \\
20 & 68.6 & 46.9 & 98.5 \\
\hline
\end{tabular}

${ }^{a}$ Values are the average of two independent experiments.

The specificity experiments demonstrated that this assay required a higher LOD for plasma samples than for sera, since plasma samples demonstrated higher non-specific activity, possibly due to cellular toxicity of the anticoagulants used for their preparation. For this reason, due to the potential for low level non-specific activity with some samples, immune monitoring of neutralizing antibodies in HIV-1 vaccine clinical trial immune monitoring assays should test, when possible, a corresponding pre-immune sample for every post-immune sample. The use of plasma samples should be avoided in the A3R5 assay whenever corresponding serum samples are available for testing. The specificity results also indicated that the use of DEAE-Dextran in the assay and the practice of heat inactivating the samples before testing them in the A3R5 assay did not affect the overall neutralization results.

Assay precision was demonstrated by successful repeatability measurements within operator and within assay. Intermediate precision was evaluated by comparing two operators, three different days of testing and three instruments. When a regression model was used to compare the differences between operators, within specific virus/sample combinations, the overall operator, instrument, or assay day effects in the model were not significant, but certain virus/sample combinations were problematic. Results from inter-laboratory precision studies indicated that the three laboratories accurately identified the sample categories (negative, low, medium, high titers). Although the results from all labs were within acceptance criteria for the negative and the low neutralization titer values, the category with the highest variability was the medium titer category, followed by the high titer category. The combinations that failed to meet the acceptance criteria always included the IBB and the W04 samples, similarly to what was observed in the intermediate precision results. Both W04 and IBB samples were collected by Blood Banks from HIV-1-seropositive donors; the W04 sample was a plasma sample and the IBB was a serum sample with an unknown preservative addition, obtained from whole blood over 25 years ago. No further information was available from the Blood Banks. These samples were utilized because of the high sample volume required to perform the intermediate precision and reproducibility experiments. It must be noted that all tests performed with the mAbs showed complete reproducibility between all operators. The failed results obtained from using the IBB and the W04 samples compared to the other samples, suggest, but do not prove, an intrinsic problem with the nature of these samples rather than a lack of assay reproducibility. Additionally, as described in the optimization experiments, the neutralizing activity of IBB and plasma samples was more sensitive to the input number of A3R5 cells/well than the mAbs (Fig. 1). Difference in the counting devices used by the operators could account for some of the variability observed in the reproducibility experiments. Interestingly, the intra-operator variability was randomly distributed between the three testing laboratories. The number of laboratories performing the A3R5 assay has recently increased to eleven, allowing for further reproducibility testing similar to the studies performed for the TZM-bl assay (Todd et al., 2012). This will be seminal to the development of a global Proficiency Testing Program for the A3R5 assay.

Accuracy of the A3R5 assay was assessed using the TZM-bl assay as the accepted "gold standard" assay for detecting neutralizing antibodies. The experiments performed to address accuracy demonstrated that the A3R5 was as accurate as the TZM-bl assay for detecting neutralizing antibody responses. Moreover, the A3R5 assay was more sensitive than the TZM-bl assay for detecting neutralization of tier 2 viruses but was equally sensitive for detecting neutralization of the tier $1 \mathrm{~A}$ virus, SF162.LucR. A similar improved sensitivity of the A3R5 assay for detecting neutralization of tier 2 but not tier 1 viruses was reported recently, and was associated with antibodies to the variable loops and CD4 binding domain of gp120, and with epitopes in the cluster I region of gp41 (deCamp et al., 2014). The higher sensitivity of the A3R5 assay makes it complementary to, but not a substitute for, the TZM-bl assay. The TZM-bl assay is adequately sensitive for studies of the neutralizing antibody response in HIV-1-infected individuals and for characterizing broadly neutralizing mAbs, where it remains the assay of choice because of its lower cost. For assessments of vaccine-elicited neutralizing antibodies, we recommend the A3R5 assay for initial assessments of tier 2 virus neutralization because of its greater sensitivity. Subsequent assays in TZM-bl cells may be performed with samples that test positive in the A3R5 assay. Vaccine sera may be assayed with tier 1 viruses only in TZM-bl cells because these viruses are equally sensitivity in both assays, and because the TZM-bl assay is lower in cost.

Neutralization curves showed linearity over an approximate range of $20-80 \%$ neutralization. Values in this range were proportional to the concentration of neutralizing antibodies in the sample.

A validated assay is one that is not easily affected by variations in physical laboratory conditions. It is important to understand the operational parameters, since possible changes in the procedure can be encountered on a daily basis. Thus, the robustness of the A3R5 assay was determined for parameters involved in the optimal number of A3R5 cells/well, the incubation time for the assay, the stability of A3R5 cells over time in culture, the integrity of the samples if exposed to freeze and thaw cycles or prolonged storage conditions, and the use of different curve fitting algorithms to calculate the neutralization titers.

\section{Conclusions}

In conclusion, as a result of the optimization and validation studies reported herein, the A3R5 assay was deemed suitable for use as an immune monitoring endpoint assay for HIV-1 vaccine clinical trials. The greater sensitivity of the A3R5 assay 
compared to the TZM-bl assay for tier 2 virus neutralization supports the use of the A3R5 assay for immune monitoring purposes. Additional information is needed to determine whether low-level neutralizing activity that is only detected in the A3R5 assay neutralization can be beneficial in a vaccine setting. Since it is currently unknown what level and breadth of neutralization in any assay will predict a significantly protective vaccine effect, we recommend that both assays continue to be used for HIV-1 vaccine development.

\section{Acknowledgments}

This study was supported in part by the NIAID, NIH, Department of Health and Human Services (Contract ID: HHSN272201000045C), by the Bill \& Melinda Gates Foundation's Collaboration for AIDS Vaccine Discovery/ Comprehensive Antibody Vaccine Immune Monitoring Consortium (CAVD/CA-VIMC) (Grant IDs: 38619 \& 1032144), by the Duke University Center for AIDS Research (CFAR), a NIH funded program (Program ID: 5P30 AI064518), Flow Cytometry Core, and by the University of Alabama at Birmingham CFAR (P30 AI027767), Virology Core. We wish to thank the EQAPOL Scientific Leadership, the EQAPOL and the CAVD/ CA-VIMC Scientific Advisory Board members for their input into the A3R5 Program; CHAVI and BSRI for providing the plasma and serum samples; M. Seaman, H. von Briesen and their laboratories for participating in the reproducibility studies; J. Hural for helpful discussions on specificity; D. Ozaki, K. Greene, H. Gao, A. Sanchez and Jennifer Kirchherr for their continued support.

\section{References}

Bonsignori, M., Hwang, K.K., Chen, X., Tsao, C.Y., Morris, L., Gray, E., Marshall, D.J., Crump, J.A., Kapiga, S.H., Sam, N.E., Sinangil, F., Pancera, M., Yongping, Y., Zhang, B., Zhu, J., Kwong, P.D., O'Dell, S., Mascola, J.R., Wu, L., Nabel, G.J., Phogat, S., Seaman, M.S., Whitesides, J.F., Moody, M.A., Kelsoe, G., Yang, X., Sodroski, J., Shaw, G.M., Montefiori, D.C., Kepler, T.B., Tomaras, G.D., Alam, S.M., Liao, H.X., Haynes, B.F., 2011. Analysis of a clonal lineage of HIV-1 envelope V2/V3 conformational epitope-specific broadly neutralizing antibodies and their inferred unmutated common ancestors. J. Virol. 85, 9998.

Bonsignori, M., Montefiori, D.C., Wu, X., Chen, X., Hwang, K.K., Tsao, C.Y., Kozink, D.M., Parks, R.J., Tomaras, G.D., Crump, J.A., Kapiga, S.H., Sam, N. E., Kwong, P.D., Kepler, T.B., Liao, H.X., Mascola, J.R., Haynes, B.F., 2012. Two distinct broadly neutralizing antibody specificities of different clonal lineages in a single HIV-1-infected donor: implications for vaccine design. J. Virol. 86, 4688.

DeCamp, A., Hraber, P., Bailer, R.T., Seaman, M.S., Ochsenbauer, C., Kappes, J., Gottardo, R., Edlefsen, P., Self, S., Tang, H., Greene, K., Gao, H., Daniell, X., Sarzotti-Kelsoe, M., Gorny, M.K., Zolla-Pazner, S., Labranche, C.C., Mascola, J.R., Korber, B.T., Montefiori, D.C., 2014. Global panel of HIV-1 Env reference strains for standardized assessments of vaccine-elicited neutralizing antibodies. J. Virol. 88 (5), 2489-2507 http://dx.doi.org/10. 1128/JVI.02853-13 (Epub 2013 Dec 18).

Edmonds, T.G., Ding, H., Yuan, X., Wei, Q., Smith, K.S., Conway, J.A., Wieczorek, L., Brown, B., Polonis, V., West, J.T., Montefiori, D.C., Kappes, J.C., Ochsenbauer, C., 2010. Replication competent molecular clones of HIV-1 expressing Renilla luciferase facilitate the analysis of antibody inhibition in PBMC. Virology 408, 1.

Ezzelle, J., Rodriguez-Chavez, I., Darden, J., Stirewalt, M., Kunwar, N., Hitchcock, R., Walter, T., D'souza, M., 2008. Guidelines on good clinical laboratory practice: bridging operations between research and clinical research laboratories. J. Pharm. Biomed. Anal. 46, 18.

Fenyo, E.M., Heath, A., Dispinseri, S., Holmes, H., Lusso, P., Zolla-Pazner, S., Donners, H., Heyndrickx, L., Alcami, J., Bongertz, V., Jassoy, C., Malnati, M., Montefiori, D., Moog, C., Morris, L., Osmanov, S., Polonis, V., Sattentau, Q., Schuitemaker, H., Sutthent, R., Wrin, T., Scarlatti, G., 2009. International network for comparison of HIV neutralization assays: the NeutNet report. PloS ONE 4, e4505.

Folks, T., Benn, S., Rabson, A., Theodore, T., Hoggan, M.D., Martin, M., Lightfoote, M., Sell, K., 1985. Characterization of a continuous T-cell line susceptible to the cytopathic effects of the acquired immunodeficiency syndrome (AIDS)associated retrovirus. Proc. Natl. Acad. Sci. U. S. A. 82, 4539.

I.C.H. Guideline, Schwager, T., 2005. Validation of Analytical Procedures: Text and Methodology Q2 (R1) (2005). Website http://www.ich.org/ fileadmin/Public_Web_Site/ICH_Products/Guidelines/Quality/Q2_R1/ Step4/Q2_R1_Guideline.pdf.

Mascola, J.R., D'Souza, P., Gilbert, P., Hahn, B.H., Haigwood, N.L., Morris, L., Petropoulos, C.J., Polonis, V.R., Sarzotti, M., Montefiori, D.C., 2005. Recommendations for the design and use of standard virus panels to assess neutralizing antibody responses elicited by candidate human immunodeficiency virus type 1 vaccines. J. Virol. 79, 10103.

McLinden, R.J.L., Chenine, C.C., A.L., Polonis, V.R., Eller, M.A., Wieczorek, L., Ochsenbauer, C., Kappes, J.C., Perfetto, S., Montefiori, D.C., Michael, N.L., Kim, J. H., 2013. Detection of HIV-1 neutralizing antibodies in a human CD4+/ CXCR4+/CCR5 + T-lymphoblastoid cell assay system. PloS 8 (11), e77756. http://dx.doi.org/10.1371/journal.pone.0077756 (Nov 28).

Montefiori, D.C., 2009. Measuring HIV neutralization in a luciferase reporter gene assay, In: Vinayaka, G.V.K., Prasad, R. (Eds.), Second edition. Methods Mol. Virol, HIV Protocols, vol. 485. Humana Press, p. 395.

Montefiori, D.C., Karnasuta, C., Huang, Y., Ahmed, H., Gilbert, P., de Souza, M. S., McLinden, R., Tovanabutra, S., Laurence-Chenine, A., Sanders-Buell, E., Moody, M.A., Bonsignori, M., Ochsenbauer, C., Kappes, J., Tang, H., Greene, K., Gao, H., LaBranche, C.C., Andrews, C., Polonis, V.R., RerksNgarm, S., Pitisuttithum, P., Nitayaphan, S., Kaewkungwal, J., Self, S.G., Berman, P.W., Francis, D., Sinangil, F., Lee, C., Tartaglia, J., Robb, M.L., Haynes, B.F., Michael, N.L., Kim, J.H., 2012. Magnitude and breadth of the neutralizing antibody response in the RV144 and Vax003 HIV-1 vaccine efficacy trials. J. Infect. Dis. 206, 431.

Ochsenbauer, C., Kappes, J.C., 2009. New virologic reagents for neutralizing antibody assays. Curr. Opin. HIV AIDS 4, 418

Ochsenbauer, C., Edmonds, T.G., Ding, H., Keele, B.F., Decker, J., Salazar, M.G., Salazar-Gonzalez, J.F., Shattock, R., Haynes, B.F., Shaw, G.M., Hahn, B.H., Kappes, J.C., 2012. Generation of transmitted/founder HIV-1 infectious molecular clones and characterization of their replication capacity in CD4 T lymphocytes and monocyte-derived macrophages. J. Virol. 86, 2715.

Ozaki, D.A., Gao, H., Todd, C.A., Greene, K.M., Montefiori, D.C., Sarzotti-Kelsoe, M. 2012. International technology transfer of a GCLP-compliant HIV-1 neutralizing antibody assay for human clinical trials. PloS ONE 7, e30963.

Piehler, B., Nelson, E.K., Eckels, J., Ramsay, S., Lum, K., Wood, B., Greene, K.M., Gao, H., Seaman, M.S., Montefiori, D.C., Igra, M., 2011. LabKey Server NAb: a tool for analyzing, visualizing and sharing results from neutralizing antibody assays. BMC Immunol. 12, 33.

Platt, E.J., Wehrly, K., Kuhmann, S.E., Chesebro, B., Kabat, D., 1998. Effects of CCR5 and CD4 cell surface concentrations on infections by macrophagetropic isolates of human immunodeficiency virus type 1 . J. Virol. 72 (1998), 2855-2864.

Polonis, V.R., Brown, B.K., Rosa Borges, A., Zolla-Pazner, S., Dimitrov, D.S., Zhang, M.Y., Barnett, S.W., Ruprecht, R.M., Scarlatti, G., Fenyo, E.M., Montefiori, D.C., McCutchan, F.E. Michael, N.L, 2008. Recent advances in the characterization of HIV-1 neutralization assays for standardized evaluation of the antibody response to infection and vaccination. Virology 375, 315.

Sarzotti-Kelsoe, M., Cox, J., Cleland, N., Denny, T., Hural, J., Needham, L., Ozaki, D., Rodriguez-Chavez, I.R., Stevens, G., Stiles, T., 2009. Evaluation and recommendations on good clinical laboratory practice guidelines for phase I-III clinical trials. PLoS Med. 6, e1000067.

Sarzotti-Kelsoe, M., Bailer, R.T., Turk, E., Lin, C.L., Bilska, M., Greene, K.M., Gao, H., Todd, C.A., Ozaki, D.A., Seaman, M.S., Mascola, J.R., Montefiori, D.C., 2013. Optimization and validation of the TZM-bl assay for standardized assessments of neutralizing antibodies against HIV-1. J. Immunol. Methods. http:// dx.doi.org/10.1016/j.jim.2013.11.022 (Dec 1. pii: S0022-1759(13)00359-1, [Epub ahead of print] PMID: 24291345 (in this issue)).

Seaman, M.S., Janes, H., Hawkins, N., Grandpre, L.E., Devoy, C., Giri, A., Coffey, R.T., Harris, L., Wood, B., Daniels, M.G., Bhattacharya, T., Lapedes, A., Polonis, V.R., McCutchan, F.E., Gilbert, P.B., Self, S.G., Korber, B.T., Montefiori, D.C., Mascola, J.R., 2010. Tiered categorization of a diverse panel of HIV-1 Env pseudoviruses for assessment of neutralizing antibodies. J. Virol. 84, 1439.

Stiles, T., Grant, V., Mawby, N., 2003. Good Clinical Laboratory Practice (GCLP): A Quality System for Laboratories That Undertake the Analyses of Samples from Clinical TrialsBARQA, Medical Laboratories. 1-18.

Todd, C.A., Greene, K.M., Yu, X., Ozaki, D.A., Gao, H., Huang, Y., Wang, M., Li, G., Brown, R., Wood, B., D'Souza, M.P., Gilbert, P., Montefiori, D.C., SarzottiKelsoe, M., 2012. Development and implementation of an international proficiency testing program for a neutralizing antibody assay for HIV-1 in TZM-bl cells. J. Immunol. Methods 375, 57-67. 
Todd, C.A., Greene, K.M., Yu, X., Ozaki, D.A., Gao, H., Huang, Y., Wang, M., Li, G. , Brown, R., Wood, B., D'Souza, M.P., Gilbert, P., Montefiori, D.C., SarzottiKelsoe, M., 2012. Development and implementation of an international proficiency testing program for a neutralizing antibody assay for HIV-1 in TZM-bl cells. J. Immunol. Methods 375, 57-67.

Todd, C.A., Sanchez, A.M., Garcia, A., Denny, T.N., Sarzotti-Kelsoe, M., 2013. Implementation of Good Clinical Laboratory Practice (GCLP) guidelines within the External Quality Assurance Program Oversight Laboratory (EQAPOL). J. Immunol. Methods. http://dx.doi.org/10.1016/j.jim.2013.09.012 (Oct 9. pii: S0022-1759(13)00268-8, [Epub ahead of print] (in this issue)).

Wei, X., Decker, J.M., Liu, H., Zhang, Z., Arani, R.B., Kilby, J.M., Saag, M.S., Wu, X., Shaw, G.M., Kappes, J.C., 2002. Emergence of resistant human immunodeficiency virus type 1 in patients receiving fusion inhibitor (T-20) monotherapy. Antimicrob. Agents Chemother. 46, 1896.
Wei, X., Decker, J.M., Wang, S., Hui, H., Kappes, J.C., Wu, X., Salazar-Gonzalez, J.F., Salazar, M.G., Kilby, J.M., Saag, M.S., Komarova, N.L., Nowak, M.A., Hahn, B.H., Kwong, P.D., Shaw, G.M., 2003. Antibody neutralization and escape by HIV-1. Nature 422, 307.

Wu, X., Zhou, T., Zhu, J., Zhang, B., Georgiev, I., Wang, C., Chen, X., Longo, N.S., Louder, M., McKee, K., O'Dell, S., Perfetto, S., Schmidt, S.D., Shi, W., Wu, L., Yang, Y., Yang, Z.Y., Yang, Z., Zhang, Z., Bonsignori, M., Crump, J.A., Kapiga, S.H., Sam, N.E., Haynes, B.F., Simek, M., Burton, D.R., Koff, W.C., Doria-Rose, N.A., Connors, M., Mullikin, J.C., Nabel, G.J., Roederer, M., Shapiro, L., Kwong, P.D., Mascola, J.R., 2011. Focused evolution of HIV-1 neutralizing antibodies revealed by structures and deep sequencing. Science 333, 1593. 